\section{Morphological changes of the anterior segment after laser peripheral iridotomy in primary angle closure}

K Lei, N Wang, L Wang and B Wang

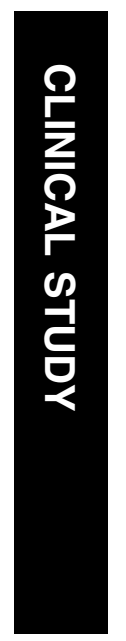

Keywords: primary angle closure; laser therapy; anterior segment; optical coherence tomography

\section{Introduction}

Primary angle-closure (PAC) glaucoma is the major form of glaucoma in Chinese population, and one of the leading causes of blindness in China. ${ }^{1,2}$ Laser peripheral iridotomy (LPI) is widely regarded as the first-line intervention for either acute or chronic types of angle-closure glaucoma in early stage..$^{3-5}$ LPI may help in reversing the appositional angle closure and control the intraocular pressure, so the development of primary angle closure (PAC) into primary angle closure glaucoma (PACG) could be prevented. ${ }^{6,7}$ The mechanism of LPI is that it creates an opening to bypass the pupillary block and, therefore, allows a convex iris to flatten and thus widens the anterior chamber (AC) angle. ${ }^{8,9}$

There are a lot of techniques that can be used to observe the anterior segment morphology, such as optical pachymetry, ultrasound biomicroscopy (UBM), A-scan ultrasound, Scheimpflug technique, and IOLMaster. A large number of studies have assessed the change of anterior segment morphology using the abovementioned techniques, and most of them believed that the peripheral anterior chamber was deepened and the anterior angle was widened significantly after LPI, whereas the central anterior chamber was not changed. ${ }^{8-16}$

However, all of the above methods might have some limitations. Either the accommodation or the illumination could not be controlled, or the examination would touch the
Tongren Eye Center, Beijing Tongren Hospital, Capital Medical University, Beijing, China

Correspondence: N Wang, Tongren Eye Center, Beijing Tongren Hospital, Capital Medical University No.1 Dongjiaominxiang Street,

Dong Cheng district, Beijing 100730,

China

Tel: + 861058269919 ; Fax: + 861058269920

E-mail: wningli@

trhos.com

Received: 13 June 2007 Accepted in revised form: 5 November 2007

Published online:

7 December 2007

Contributions: Design of the study (Kun Lei, Ningli Wang); conduct of the study (Kun Lei, Lan Wang, Bingsong Wang); analysis and interpretation (Kun Lei, Ningli Wang). 
eyes, thereby causing some measuring errors. Anterior segment optical coherence tomography (AS-OCT), which allows rapid and noncontact examination, gives highresolution images of anterior segment. During examination, a subject fixates on an optical target whose focus can be adjusted to compensate for the subject's spherical ametropia, and images of the unaccommodated eye can be acquired. ${ }^{17,18}$ Therefore, we used the AS-OCT to find out the changes of anterior segment morphology after LPI in PAC eyes.

\section{Patients and methods}

Consecutive patients with PAC attending the clinics of two glaucoma specialists in Tongren Eye Centre from March 2006 to September 2006 were included in the present study. We included only the local patients, because they were easy to follow up. Written informed consent was obtained from all patients, approval was taken from the ethics committee of the Tongren Eye Centre, and the study was performed according to the tenets of the Declaration of Helsinki. Slit-lamp biomicroscope, ophthalmoscopy, gonioscopy, visual field testing, and intraocular pressure (IOP) measurement were done on these patients. The enrolled eye had gonioscopically documented presence of peripheral anterior synechiae (PAS) or elevated IOP in an occludable angle with the trabecular meshwork not being visualized in more than $270^{\circ}$ of the angle. The visual field and the optic disc appearance were normal. ${ }^{19}$ We excluded the eyes that once had acute attack, and eyes that had surgeries or laser treatments were not enrolled as well. Pilocarpine had not been used for at least 7 days, and other antiglaucoma drugs (topical and systemic) were stopped at least 3 days before AS-OCT examination.

AS-OCT and Goldmann applanation tonometer were performed sequently on each selected eye. AS-OCT (Carl Zeiss Meditec, Dublin, CA, USA, Visante ${ }^{\mathrm{TM}}$ OCT) uses a $1310 \mathrm{~nm}$ wavelength to scan at a rate of eight frames per second. The scan depth is $6 \mathrm{~mm}$ and the width is $16 \mathrm{~mm}$. The axial and lateral resolution is 25 and $60 \mu \mathrm{m}$, respectively. During the examination, the patient was seated comfortably and positioned in a chinrest similar to what was typically used for a slit lamp. To perform ASOCT imaging in a nonaccommodated state, the patient's refractive correction was used to adjust the internal fixation target for the patient's distance correction. Images of his anterior chamber were obtained in a few seconds. As there was no contact, there was no undue pressure on the eye. The AS-OCT imaging was performed under uniform conditions of illumination in a room. The room illumination was $44 \pm 0.5$ lux by a light metre (TES-1330A, Taiwan). All of the measurements were performed by a well-trained ophthalmologist. Each examination was carried out at the same time of the day before and after laser peripheral iridotomy (LPI) for each patient. The time period between LPI and post-LPI examination was 7 days at least.

We used the anterior segment single-line scan mode to scan the tomographical cross-section of the anterior chamber in the horizontal meridian for three times, and the same mode was used to acquire the lens images for three times. We acquired 15 images in each scan, and the one with the best quality was saved. The chosen images should have reflection axis of the light source, which was an optically produced, vertical white line through the centre of the cornea. After images were stored, the measurements were done using the built-in software (version 1.0) by a trained ophthalmologist. Qualitative analysis was used to assess the extent of iris bowing and the change in angle after LPI. The line joining the root of the iris and the pupillary margin of the iris was used as a fixed reference. Iris bowing existed when the posterior surface of iris was before this line. Angle closure was defined by AS-OCT as contact between the peripheral iris and angle wall anterior to the scleral spur.

Parameters including central anterior chamber depth (cACD), pupil diameter (PD), and lens thickness (LT) were analysed quantitatively. The cACD was calculated as the distance from the posterior surface of the cornea to the anterior surface of the lens along with the perpendicular bisector of the anterior chamber horizontal diameter, which was a line with its two endpoints placed on each scleral spur in the horizontal image. PD was measured along the horizontal direction. The distance from the anterior surface to the posterior surface of the lens along the reflection axis of the light source was termed as LT. We performed one measurement in each of the three obtained images, and the average of the measurements was used as the final value.

To calculate the anterior chamber volume (ACV), we carried out the anterior segment quad line scan mode for three times to obtain 12 cross-sectional images of the AC at $15^{\circ}$ intervals. The 12 obtained images were input in a computer and processed using a specialized software designed by Wang et al. ${ }^{20}$ First, we outlined the ACs and marked the geometric axes of the images manually, then the volume of every $15^{\circ}$ meridional section can be approximated by the sums of the partial volumes of a series of concentric cylindrical sleeves. Finally, the sum of the volumes of the 12 images, which expresses the value of the ACV, was computed. The last two steps can be quickly carried out with the aid of the specialized programme.

Consecutive argon and Nd:YAG (neodymiumyttrium-aluminium-garnet) LPIs were performed in each patient with the following settings: Argon- $100 \mu \mathrm{m}$, $0.1 \mathrm{~s}, 0.8 \mathrm{~mJ}, 10-20$ burns followed by Nd:YAG $5-8 \mathrm{~mJ}$, 
1-5 shots. All patients received $0.1 \%$ dexamethasone and $0.3 \%$ tobramycin eye drops four times a day for 3 days. $0.5 \%$ timolol eye drops were applied twice a day for 2 or 3 days on the eye, if the IOP was higher than $21 \mathrm{mmHg}$ measured $1 \mathrm{~h}$ after LPI.

Statistical analyses were performed using SPSS for Windows version 11.5. Changes in mean values before and after LPI were assessed using Wilcoxon signed-rank test. The correlation between the CACD and the ACV at the baseline was determined by Pearson's correlation coefficient. A $P$-value of less than 0.05 was considered statistically significant. If both eyes met the criterion, only the parametres of the right eyes were analysed.

\section{Results}

Fifteen eyes of 15 patients with PAC were enrolled in the present study. The mean age was $66.0 \pm 5.7$ years (range: 55-74 years). There were 4 male and 11 female patients. The average time period from LPI to reexamination was $20.2 \pm 12.7$ days (range: 7-48 days). All follow-up patients did not use any topical or systemic drugs. PAS was detected in 9 out of 15 eyes in gonioscopy. The average clock hours of PAS was $1.3 \pm 1.4(0-4)$ clock hours.

In horizontal AS-OCT images, ten eyes were found to have appositional angle closure in nasal, temporal or both quadrants before LPI. After LPI, all of them reopened and the width of the other angles increased. The iris changed from convexity to flattening in all eyes. Figure 1 shows the AS-OCT images of the AC of one patient before and after LPI. The posterior surface of the iris flattened along with the significantly widening angle after LPI.

Table 1 shows changes in those parametres with their statistical significance.

In all cases but two, the cACD increased after LPI. There were eight eyes (54\%) increased by $10-40 \mu \mathrm{m}$ and five eyes (33\%) increased by $50-80 \mu \mathrm{m}$. The ACV of all eyes increased.
In these two exceptions, a decrease in CACD of 13 and $23 \mu \mathrm{m}$, representing a decrease of $0.80-1.22 \%$ of the original depth, was found after LPI. After further analysis of the two patients, we have not found any clinical features to differentiate them from others. The detailed AS-OCT parameters of the two patients showed that the CACD of one patient decreased by $13 \mu \mathrm{m}$, as well as the lens thickened by $13 \mu \mathrm{m}$, and the PD had decreased by $0.743 \mathrm{~mm}$ after LPI. The lens of the other patient, whose cACD decreased by $23 \mu \mathrm{m}$, was so thick that it was out of the range of AS-OCT measurement (the lens might be thicker than $6.0 \mathrm{~mm}$ ).

A statistical correlation was found between the CACD and the ACV at the baseline. The correlation coefficient was $0.928(P=0.000)$ and $R^{2}=0.86$.

\section{Discussion}

We studied the morphology of the anterior segment of PAC qualitatively and quantitatively before and after LPI using AS-OCT and found the iris contours of all the examined eyes were convex before LPI; however, none showed convex contours anymore after LPI. All anterior angles widened after LPI. These changes were consistent with previous reports analysing quantitatively. ${ }^{8-16}$ We did not measure the angle opening distance (AOD), because the software in AS-OCT only includes a measuring system capable of calculating distance and angle.

In PAC eyes, most of whose angles were appositionally closed, LPI was an effective therapy, as it made a hole in the iris, allowing aqueous to flow directly from the posterior to the anterior chamber, bypassing the pupillary block. This caused an equalization of the pressure gradient across the iris and allows the iris to flatten and fall posteriorly from the peripheral angle.

It has been proved by many authors that the pACD deepens after LPI; however, whether the cACD changes is uncertain. No change in CACD was reported by several
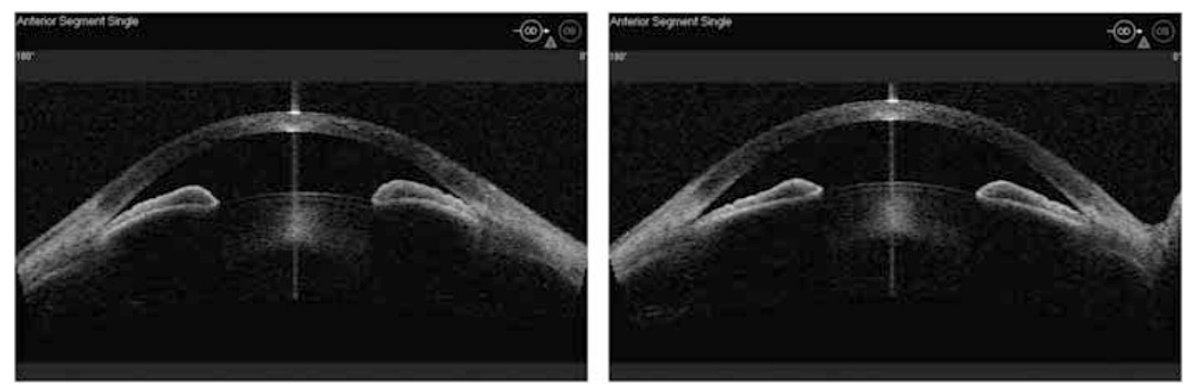

Figure 1 Anterior segment optical coherence tomography (AS-OCT) images of a 65-year-old woman. (Left) The woman's anterior chamber is shown with an appositional angle-closure and iris bowing before LPI. Right panel: after LPI the iris flattens and the chamber angle opens. 
Table 1 The anterior chamber parametres before and after LPI in PAC

\begin{tabular}{lccccc}
\hline & $c A C D(\mathrm{~mm})$ & $P D(\mathrm{~mm})$ & $L T(\mathrm{~mm})^{\mathrm{a}}$ & $A C V(\mu l)$ & $I O P(\mathrm{mmHg})$ \\
\hline Pre-LPI & $1.939 \pm 0.228$ & $4.229 \pm 0.579$ & $5.152 \pm 0.237$ & $73.86 \pm 14.58$ & $17.8 \pm 3.3$ \\
& $(1.583-2.450)$ & $(3.337-5.313)$ & $(4.710-5.690)$ & $(52.11-108.22)$ & $(12.0-22.7)$ \\
Post-LPI & $1.970 \pm 0.235$ & $4.133 \pm 0.566$ & $5.146 \pm 0.240$ & $84.14 \pm 17.45$ & $15.9 \pm 3.1$ \\
& $(1.607-2.513)$ & $(3.157-5.300)$ & $(4.703-5.697)$ & $(54.35-124.89)$ & $(8.7-20)$ \\
$P$ & 0.003 & 0.233 & 0.238 & 0.001 & 0.043 \\
\hline
\end{tabular}

Given as mean \pm SD (range) with $P$-values for the difference between pre-LPI and post-LPI.

$\mathrm{LPI}=$ laser peripheral iridotomy; $\mathrm{PAC}=$ primary angle closure; $\mathrm{cACD}=$ central anterior chamber depth; $\mathrm{PD}=$ pupil diameter; $\mathrm{LT}=$ lens thickness; $\mathrm{ACV}=$ anterior chamber volume; IOP $=$ intraocular pressure.

${ }^{a}$ There was a patient whose lens was too thick to be measured, so the results of the other 14 eyes were analysed.

researches using different techniques from slit lamp to UBM. $8,9,12-14$

Gazzard et al $^{16}$ studied the effect of LPI on 55 fellow eyes of acute PACG patients. Although the cACD did not change significantly using optical pachymetry, the result of A-scan showed the CACD increased significantly after LPI. The authors ascribed the opposite result to the error caused by A-scan, because the probe of which might compress the eye.

In our study, a mean increase in cACD of $31 \mu \mathrm{m}$, representing an increase of $1.6 \%$ of the original cACD, was found after LPI. Different from most other studies, the change was statistically significant. The difference in methods for measurement may play a role in it.

The most common method for ACD measurement is ultrasonography, which may result in inaccurate values caused by indentation of the cornea and shallowing of the anterior chamber with the probe tip of the US device. Although indentation of the cornea during contact measurements can be eliminated with the immersion technique, off-axis measurement might produce another source of error during measurement. Nemeth et al ${ }^{21}$ have proved that the intraobserver repeatability was better with AS-OCT than with immersion A-scan As for optical methods such as pachymetry, IOLMaster, and Scheimpflug photography, which are noncontact as well, their results are likely to be affected by accommodation and illumination. The newly developed equipment AS-OCT, using $1310 \mathrm{~nm}$ infrared light, adapts technology that has been used for the posterior segment to the anterior segment. The AS-OCT allows rapid, noncontact examination, and the accommodation can be well controlled. The error in position of scan could be reduced by proper alignment of eyes.

Dada $e t a l^{22}$ performed UBM on 54 PAC eyes and found that the cACD increased significantly after LPI in consistent with our study. But their result showed that the cACD increased by $110 \mu \mathrm{m}$, which was far more than that observed in our study $(31 \mu \mathrm{m})$. The difference in the results might probably rest with the difference in the two measuring methods. A gel or water bath has to be placed between the probe and the eye when the UBM is performed. These methods might lead to slight anterior segment distortion through external compression. The patient is supine when doing UBM but seated when ASOCT is performed, and the difference in position would affect the result too. In addition, our study had more restriction on the drugs topically or systemically, and this may cause difference in the results between the two studies as well.

The exact mechanism for deepening of the central AC is not clear. Dada et $a l^{22}$ hypothesized that the obstructed aqueous might find its way into the vitreous and push the lens forward. Decompression of the aqueous with relief of the pupillary block may thus prevent this aberrant aqueous movement, relieve the forward pressure on the lens, and result in a deepening of the central anterior chamber. Quigley et $a l^{23}$ thought that the lens was subjected to the posterior chamber pressure over its posterior surface and over all of its anterior surface, except that portion in the pupil-for which the lower anterior chamber ambient pressure is operative. Iridotomy would eliminate any differential pressure exerted on the lens (the pupillary zone would have equal pressure to that in the posterior chamber), which would perhaps allow a slight posterior movement.

To study why the cACD increased, we discussed the action of forces on the lens. We found out that the lens might be subjected to three major forces, the pressure from posterior chamber $\left(P_{\mathrm{PC}}\right)$, the pressure from anterior chamber $\left(P_{\mathrm{AC}}\right)$, and the pupillary-blocking force $(\mathrm{PBF})$ before LPI as shown in Figure 2. The summation of $P_{\mathrm{AC}}$ and $\mathrm{PBF}$ is equal to $P_{\mathrm{PC}}$, and the composition of the forces is zero so the lens is still. After the LPI makes a conduit on the iris, which allows aqueous to flow directly from the posterior to the anterior chamber, the pressure in anterior chamber then becomes equal to the one in the posterior chamber. However, the PBF still exists. The composition of forces on the lens is not zero anymore, and the balance is broken down. The PBF, pointing to the posterior, will push the lens backward. While the lens is moving backwards, it is dragged by the zonules as well. 


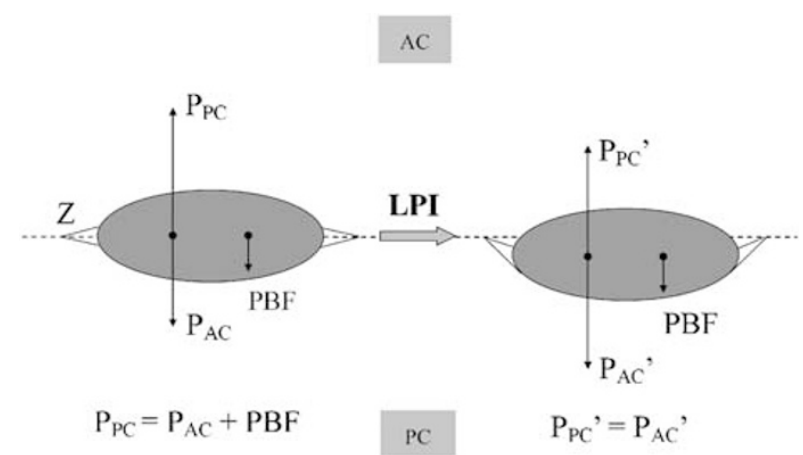

Figure 2 A sketch map showing a hypothesis of forces on lens before and after LPI. The direction and length of the lines indicate the direction and magnitude of forces on the lens. Left: The lens is in a balance when the sum of $P_{\mathrm{AC}}$ and $\mathrm{PBF}$ is equal to the $P_{\mathrm{PC}}$ before LPI. Right: after LPI, the $P_{\mathrm{AC}}$ is getting equal to the $P_{\mathrm{PC}}$ with PBF still existing, and the lens moves back. A new balance is built, because the lens is being dragged by the zonule while moving back. AC, anterior chamber; PC, posterior chamber; $P_{\mathrm{PC}}$, pressure from posterior chamber; $P_{\mathrm{AC}}$, pressure from anterior chamber; $P_{\mathrm{PC}}{ }^{\prime}$, pressure from posterior chamber after LPI; $P_{\mathrm{AC}}{ }^{\prime}$, pressure from anterior chamber after LPI; PBF papillary-blocking force; LPI, laser peripheral iridotomy; $\mathrm{Z}$, zonule.

When the lens moves back to a certain distance, it will stop and restore in a new balance, because the traction from the zonules is increasing, as the lens is moving back, and then it becomes identical to the PBF. We hypothesize that the distance the lens moved backward (the extent the cACD deepened) depends largely on two factors, the PBF and the traction of the zonules.

In addition, there were two cases whose cACD decreased after LPI, but we could not differentiate the two patients from others by symptoms and physical examinations. The cACD of one patient decreased while the LT increased the same amount, and the PD decreased 14 days after LPI. We considered this might be due to the residual action of pilocarpine administered when LPI was being performed, which cause contraction of the ciliary body and thickening of the lens.

To date, we have not found any report about the change of LT and PD after LPI. The LT and PD did not change statistically after LPI in this study.

The ACV was calculated by 12 coaxial cross-sectional images of the anterior chamber, using a specialized software as described previously. The mean ACV increased from $73.86 \pm 14.58$ to $84.14 \pm 17.45 \mu \mathrm{l}$ after LPI, increasing by $13.9 \%$, and this was in accordance with the previous studies, although we used different methods. ${ }^{24-26}$ The increment in $\mathrm{CACD}(31 \mu \mathrm{m})$ will contribute to the increase of the ACV by not more than $1 \mu \mathrm{l}$ by estimation, so the great change in ACV was mostly because of the flattening of iris shape after LPI. ${ }^{25}$ Rabsilber et $a l^{27}$ proved that the ACV was in correlation with the CACD using
Scheimpflug photography. The strong correlation between the two parametres was also found in our study, indicating that the shallower the central $\mathrm{AC}$ was the smaller was the ACV.

Without the use of any ocular hypotensive medicine, the IOP decreased by $1.9 \mathrm{mmHg}$ after LPI. The reduction in IOP may be due to the reopening of angles that were appositionally closed before LPI, and the drainage function of these angles were still normal.

In summary, this study establishes that LPI not only leads to increases in pACD and ACV, but also to an increase in CACD. A long-term follow-up of these PAC patients is needed to confirm the long-term effect of LPI on PAC eyes. AS-OCT is a promising method for accurate anterior segment biometry, but the software is still in development and requires manual measurement using the software's markers. Improvements to the software of AC-OCT and image resolution are underway.

\section{Acknowledgements}

The authors have no commercial interest in any of the material discussed in the article.

\section{References}

1 Quigley HA. Number of people with glaucoma worldwide. Br J Ophthalmol 1996; 80: 389-393.

2 Foster PJ. Glaucoma in China. How big is the problem? Br J Ophthalmol 2001; 85: 1277-1282.

3 Saw SM, Gazzard G, Friedman DS. Interventions for angleclosure glaucoma: an evidence-based update. Ophthalmology 2003; 110: 1869-1878; quiz 1878-1879, 1930.

4 Nolan WP, Foster PJ, Devereux JG, Uranchimeg D, Johnson GJ, Baasanhu J. YAG laser iridotomy treatment for primary angle closure in east Asian eyes. Br J Ophthalmol 2000; 84: 1255-1259.

5 Hsiao CH, Hsu CT, Shen SC, Chen HS. Mid-term follow-up of Nd:YAG laser iridotomy in Asian eyes. Ophthalmic Surg Laser Imaging 2003; 34: 291-298.

6 McGalliard JN, Wishart PK. The effect of Nd: YAG iridotomy on intraocular pressure in hypertensive eyes with shallow anterior chambers. Eye 1990; 4: 823-829.

7 Schwartz LW, Rodrigues MM, Spaeth GL, Streeten B, Douglas C. Argon laser iridotomy in the treatment of patients with primary angle closure or pupillary block glaucoma: a clinicopathologic study. Ophthalmology 1978; 85: 294-309.

8 Jin JC, Anderson DR. The effect of iridotomy on iris contour. Am J Ophthalmol 1990; 110: 260-263.

9 Marraffa M, Marchini G, Pagliarusco A, Perfetti S, Toscano A, Brunelli C et al. Ultrasound biomicroscopy and corneal endothelium in Nd:YAG-laser iridotomy. Ophthalmic Surg Lasers 1995; 26: 519-523.

10 Yang M, Aung T, Husain R, Chan YH, Lim LS, Seah SK et al. Choroidal expansion as a mechanism for acute primary angle closure: an investigation into the change of biometric parameters in the first 2 weeks. Br J Ophthalmol 2005; 89: 288-290. 
11 Yoon KC, Won LD, Cho HJ, Yang KJ. Biomicroscopic changes after laser iridotomy or trabeculectomy in angleclosure glaucoma. Korean J Ophthalmol 2004; 18: 9-14.

12 Caronia RM, Liebmann JM, Stegman Z, Sokol J, Ritch R. Increase in iris-lens contact after laser iridotomy for pupillary block angle. Am J Ophthalmol 1996; 122: 53-57.

13 Kashiwagi K, Abe K, Tsukahara S. Quantitative evaluation of changes in anterior segment biometry by peripheral laser iridotomy using newly developed scanning peripheral anterior chamber depth analyser. Br J Ophthalmol 2004; 88: 1036-1041.

14 Jacobs IH, Krohn DL. Central anterior chamber depth after laser iridectomy. Am J Ophthalmol 1980; 89: 865-867.

15 He M, Friedman DS, Ge J, Huang W, Jin C, Lee PS et al. Laser peripheral iridotomy in primary angle-closure suspects: biometric and gonioscopic outcomes the Liwan eye study. Ophthalmology 2007; 114: 494-500.

16 Gazzard G, Friedman DS. A prospective ultrasound biomicroscopy evaluation of changes in anterior segment morphology after laser iridotomy in Asian eyes. Ophthalmology 2003; 110: 630-638.

17 Baikoff G, Lutun E, Ferraz C, Wei J. Static and dynamic analysis of the anterior segment with optical coherence tomography. J Cataract Refract Surg 2004; 30: 1843-1850.

18 Baikoff G, Lutun E, Wei J, Ferraz G. Anterior chamber optical coherence tomography study of human natural accommodation in a 19-year-old albino. J Cataract Refract Surg 2004; 30: 696-701.

19 Foster PJ, Buhrmann R, Quigley HA, Johnson GJ. The definition and classification of glaucoma in prevalence surveys. Br J Ophthalmol 2002; 86: 238-242.
20 Wang NL, Wang BS, Zhai G, Lei K, Wang L, Congdon NG. A method of measuring anterior chamber volume using the anterior segment optical coherence tomographer and specialized software. Am J Ophthalmol 2007; 143: 879-881.

21 Nemeth G, Vajas A, Tsorbatzoglou A, Kolozsvari B, Modis Jr L, Berta A. Assessment and reproducibility of anterior chamber depth measurement with anterior segment optical coherence tomography compared with immersion ultrasonography. J Cataract Refract Surg 2007; 33: 443-447.

22 Dada T, Mohan S, Sihota R, Gupta R, Gupta V, Pandey RM. Comparison of ultrasound biomicroscopic parameters after laser iridotomy in eyes with primary angle closure and primary angle closure glaucoma. Eye 2007; 21: 956-961.

23 Quigley HA, Friedman DS, Congdon NG. Possible mechanisms of primary angle-closure and malignant glaucoma. J Glaucoma 2003; 12: 167-180.

24 Lee DA, Brubaker RF, Ilstrup DM. Anterior chamber dimensions in patients with narrow angles and angleclosure glaucoma. Arch Ophthalmol 1984; 102: 46-50.

25 Coakes RL, Lloyd-Jones D, Hitchings RA. Anterior chamber volume. Its measurement and clinical application. Trans Ophthalmol Soc UK 1979; 99: 78-81.

26 Oka N, Otori Y, Okada M, Miki A, Maeda N, Tano Y. Clinical study of anterior ocular segment topography in angle-closure glaucoma using the three dimensional anterior segment analyzer Pentacam. Nippon Ganka Gakkai Zasshi 2006; 110: 398-403.

27 Rabsilber TM, Khoramnia R, Auffarth GU. Anterior chamber measurements using Pentacam rotating Scheimpflug camera. J Cataract Refract Surg 2006; 32: 456-459. 